International Journal of Medical Science and Clinical Research Studies

ISSN(print): 2767-8326, ISSN(online): 2767-8342

Volume 02 Issue 02 February 2022

Page No: 92-98

DOI: https://doi.org/10.47191/ijmscrs/v2-i2-03, Impact Factor: 5.276

\title{
Knowledge, Attitude and Perception Regarding COVID-19 Infection Control: A Cross-Sectional Study among Dentists in Bangalore City, India
}

\author{
Dr Manjusha P. Chandran ${ }^{1}$, Dr Pramila M. ${ }^{2}$, Dr Geetha S. ${ }^{3}$, Dr Akshay V. ${ }^{4}$, Dr Aswini Madhavan ${ }^{5}$ \\ 1, 2, 3,4,5 Department of Public Health Dentistry, M. R. Ambedkar Dental College and Hospital, Bangalore, India
}

\begin{abstract}
Objectives: The dental profession is one of the occupations at the highest risk of SARS-CoV-2 infection because of the involvement of aerosol-generating procedures. The aim of this study was to assess the knowledge, attitude, and perception of dentists regarding COVID19 infection control in Bangalore city.

Methods: A cross-sectional study was conducted among dentists in Bangalore city using an online questionnaire. The questions were related to socio-demographic data and the knowledge, attitude and perceptions of the dentists towards COVID-19 and infection control during dental practice. A sample size of 254 dentists was obtained after duration of 2 months. Descriptive statistics were performed and the data obtained were presented in the form of graphs and tables

Results: The study included 254 participants (188 females and 66 males) majority of whom belonged to an age group of $<30$ years $(78.3 \%)$. A total of $209(83.3 \%)$ of the study participants have completed a master's degree in dentistry. Among 254 dentists, 141 (55.5\%) of them had received training regarding infection control in dentistry while only $102(40.2 \%)$ of them had attended any training regarding COVID-19. Majority of the dentists were aware about the symptoms, modes of transmission, diagnosis, risk identification and important measures for prevention of COVID-19 transmission. Most of the dentists perceived COVID-19 as a serious public health issue $(85.4 \%)$.

Conclusions: The study has shown an acceptable level of awareness and preparedness among the dentists working in Bangalore city considering the aim of the study. However, SARS-CoV-2 infection is a novel disease and is yet to be fully explored. Adequate research has to be carried out to shed light on the unexplored areas regarding COVID-19 and infection control.
\end{abstract}

KEYWORDS: COVID-19, Dentist, Infection, Infection control

ARTICLE DETAILS

Published On:

10 February 2022

\section{INTRODUCTION}

The coronavirus disease (COVID-19) is a rapidly spreading viral infection. It is considered more contagious than severe acute respiratory syndrome (SARS) and Middle East respiratory syndrome (MERS). ${ }^{1}$ The etiological agent for COVID-19 is a novel coronavirus called severe acute respiratory syndrome coronavirus 2 (SARS-CoV-2). It causes pneumonia, with symptoms ranging from mild to deadly. SARS-CoV-2 infection in humans can cause an acute inflammatory response (cytokine storm) and respiratory failure. ${ }^{2}$ the routes of human-to-human transmission of corona virus are through airborne droplets, touching or coming in contact with an infected person or a contaminated surface. ${ }^{1}$ Virus-containing airborne droplets

92 Volume 02 Issue 02 February 2022
(5-12 micrometers) and aerosols $(5 \mathrm{~mm})$ from infected people are transmitted into the environment via breathing, speaking, coughing, or sneezing. Susceptible people can become infected if these respiratory droplets or aerosols settle on their mucosal membrane or are inhaled. It can even spread when a susceptible person touches the contaminated surface with viruses and then transfer it to their mucus membrane. ${ }^{3}$ Other routes such as blood or saliva have not been confirmed but may be possible because of the documented transmission of infectious diseases such as HIV/AIDS, hepatitis B and C virus through the same. This hence increases the concern over the route of transmission for COVID-19 in the dental setting. ${ }^{1}$ 
On January 30, 2020, the World Health Organization (WHO) declared the Coronavirus outbreak as a global health emergency of international concern and on March 11, 2020 as a global pandemic. ${ }^{4}$ As of July 6, 2020, COVID-19 had spread to 216 countries, resulting in $11,496,926$ confirmed cases and over 535,390 mortalities. ${ }^{2}$

The major concern in a dental set up during the COVID19 scenario is the risk of cross-infection between the dentists and the patients. Treatment which involves the use of rotary dental and surgical instruments such as hand pieces or ultrasonic scalers, air-water syringes and other procedures serve as a direct route for virus spread. Hence the standard protective measures taken during routine dental work may not be effective enough to halt the potential spread of coronavirus, especially during emission of large number of droplets and aerosols from asymptomatic cases. ${ }^{5}$

The SARS-CoV-2 infection has brought about a new, unanticipated challenge to dental professionals all around the world. ${ }^{6}$ Due to the involvement of aerosol generating procedures the dental profession is regarded as one of the occupations at the highest risk of SARS-CoV-2 infection. Therefore, it is critical that the risk of transmission through dental procedures is minimized through proper understanding and actions. ${ }^{2}$

In view of growing importance of Infection control in dental settings during COVID19 pandemic, it is imperative to study the awareness of dentists about the characteristics of SARS-CoV-2 and the new infection control standards. The aim of the study is to explore the knowledge, attitude and perception of dentists towards COVID19 Infection control in Bangalore city.

\section{METHODS}

\section{Study population and setting:}

A cross-sectional study was conducted to assess the knowledge, attitude and perception of dentists regarding COVID19 infection control in Bangalore City. The registered dentists who were currently working in Bangalore in private clinics, public sector or institutions and consented for participation were included in the study. The dentists who were not practicing currently were excluded from the study.

\section{Study instrument}

The questionnaire used for the survey was a structured multiple choice questionnaire in English language. It comprised of a series of questions related to sociodemographic data and the knowledge, attitude and perceptions of the dentists towards COVID-19 and infection control during dental practice. ${ }^{1}$

\section{Collection of data}

Data was collected using an online questionnaire created with the help of Google Forms. The questionnaire was sent to all the participants in various online groups for dentists in
Bangalore city using different online platforms. An online informed consent was obtained from the participants before the study after explaining the objectives and methodology of the study. Those dentists who filled the questionnaire in the allotted time period of the study were included in the study. A sample size of 254 dentists was obtained after the study duration of 2 months. Each participant was contacted individually through offline or online method to ensure that he/she was a dentist by profession and was currently working in Bangalore city.

\section{Statistical analysis of data}

The following statistical procedures were carried out: Data obtained was compiled systemically in Microsoft Excel 2013 spread sheet and a master table was prepared. The data set was subdivided and distributed meaningfully. The data was proof read, and later presented in the form of graphs and tables. Descriptive Statistical analyses were performed using Statistical Package for Social Sciences software (SPSS version 22).

\section{RESULTS}

The present study was conducted to assess the knowledge, attitude and perception of dentists regarding COVID-19 infection control in Bangalore City.

\section{Characteristics of the study population:}

The study included a total of 254 participants who filled the questionnaire within the allotted time period for data collection. The participant characteristics are given in Table 1.

A total of $209(82.3 \%)$ of the study participants have completed a master's degree in dentistry. Out of 254 dentists, $141(55.5 \%)$ of the participants had received training regarding infection control in dentistry while only $102(40.2 \%)$ of them had attended any training regarding COVID-19.

Knowledge about the symptoms, and mode of transmission of the COVID-19 Infection:

Majority of the study participants (98\%) opted fever and cough as the major symptoms followed by shortness of breath $(96.1 \%)$ and sore throat $(95.3 \%)$. Most of the dentists correctly reported the modes of transmission of COVID-19 Infection [Table 2]

Awareness about the diagnosis and risk of COVID-19 Infection and the measures for preventing its transmission in dental clinics:

Majority of the study participants $(97.6 \%)$ recorded Realtime Polymerase Chain Reaction with respiratory material as the test to be performed for diagnosis of COVID-19 Infection. [Table 3]

Most of the participants (94.9\%) considered the presence of symptoms of a respiratory infection to identify patients at risk while over $85 \%$ of the population considered history of 
travel as well as history of contact with an infected person in identification of the same in contracting COVID-19 Infection. Over $90 \%$ of the dentists opted people with immune system deficiency, elderly, people with chronic diseases and health care providers as high risk groups for COVID-19 Infection [Table 3]

Most of the dentists recorded that frequently cleaning hands using alcohol- based hand rub or soap and water, facemask usage by a known or suspected patient, routine cleaning and disinfecting of surfaces in contact with known or suspected persons and usage of Personal Protective Equipment by all health care members as the measures to be taken for prevention of COVID-19 transmission. [Table 3]

Majority of the study participants $(88.6 \%)$ knew how to use Personal Protective Equipment (PPE) and perform isolation procedures $(85 \%)$ on patients to minimize chances of exposure. $96.9 \%$ of the study participants considered Personal Protective Equipment as useful in protection from COVID-19 and also considered changing both masks and gloves regularly as very important $(93.7 \%)$ to decrease the possibility of transmitting infections to patients and themselves.

Out of 254 participants, 104 dentists (41\%) were confident in handling suspected COVID-19 patients to a considerable or great extent.

\section{Sources of information}

Approximately $77 \%$ of the study participants reported that they were up to date on the latest information about COVID19. [Table 4]

\section{Risk perception and attitude of dentists regarding COVID-19 Infection.}

Almost all the study participants perceived COVID-19 Infection as dangerous (98.4\%) [Figure 1]. Majority of the study participants $(85.4 \%)$ believed that COVID-19 is a current serious public health issue [Figure 2] and all the participants considered educating people about COVID-19 as important to prevent the spread of the diseases.

Out of 254 participants, $171(67.3 \%)$ believed that COVID19 symptoms often resolve with time and do not require any special treatment. More than half $(57.1 \%)$ of the 254 study participants preferred to avoid working with a patient who is a suspect of COVID-19.

In case a patient was sneezing or coughing in the clinic, 50.4 $\%$ of the dentists recorded that they would refer the patient without treating him/her, $46.5 \%$ recorded that they would treat the patient and ask him/her to go to the hospital and, only $3.1 \%$ recorded they would refuse the treatment and ask the patient to leave the dental clinic.

A vast majority of the dentists (99.2\%) believed that asking patients to sit far from each other, wearing masks while in the waiting room, and washing hands before getting in the dental chair as necessary and would help to decrease disease transmission.

94 Volume 02 Issue 02 February 2022
Over $80 \%$ of the study participants $(89 \%)$ recorded that they would not allow any of their dental staff to work with patients who had flu- like symptoms and considered the dentist role in teaching others about COVID-19 as very significant $(81.9 \%)$.

\section{Preparedness for COVID-19 Infection}

Majority $(73.6 \%)$ of the study participants considered themselves prepared for the COVID-19 outbreak. [Figure 3] Majority of the study participants recorded that they knew whom to contact when a situation of unprotected exposure to the virus occurs $(91.7 \%)$ and knew what to do if they have signs or symptoms of the disease $(97.2 \%)$.

\section{DISCUSSION}

This cross-sectional study provides an insight on the level of knowledge, attitude and perception of dentists working in Bangalore city on COVID-19 infection control. The survey showed that majority of the dentists belonged to a younger age group of less than 30 years $(78.3 \%)$ and had less than 5 years of dental practice $(79.9 \%)$. Most of the participants in the present study were females $(74 \%)$ considering the gender wise distribution. This may be because of the high proportion of females in dental profession in the country. This was comparable with a study done by Khader et al in 2020. ${ }^{1}$

Present study showed that majority of the study participants (50.4\%) worked in a private clinic. This result was comparable with a study done by Dhanya et al in $2016 .^{7}$

Knowledge about COVID-19 Infection was found to be adequate among the dentists in the current study. Majority of the study participants were able to identify the main symptoms of COVID-19 and the modes of transmission which could help them to recognize the threat and take appropriate actions which can be considered important in the management and control of the spread of the disease. This result was comparable with a study done in Jordon by Khader ET al. ${ }^{1}$

Majority of the study participants were aware about the diagnosis and risk of COVID-19 Infection and the measures to be taken for the prevention of its transmission in dental clinics. Almost all of the study participants (97.6\%) reported that Real-time Polymerase Reaction with respiratory material is the test to be performed for the diagnosis of COVID-19 Infection. Majority of the dentists recorded presence of symptoms of a respiratory infection, history of travel, and history of contact with an infected person to identify patients at risk of COVID-19 Infection. In a previous study done in Indonesia it was reported that $67 \%$ of the participants had comprehensive knowledge about virus detection methods. ${ }^{2}$

According to the present study, high risk groups for the disease were identified to be people with immune system deficiency, elderly people with chronic diseases and health care providers. 
Dentists in the present study were able to identify the measures to be taken in the prevention of COVID-19 transmission. Majority of the study participants recorded that disinfection and sanitation procedures and use of Personal Protective Equipment by the dental staff and patients as measures to be taken for preventing COVID-19 spread. Most of the participants agreed that the Personal Protective Equipment were useful in COVID-19 protection and knew how to use them. Most of the dentists in the current study knew how to perform isolation procedures on patients to reduce the risk of exposure and also understood the importance of timely changing of protective barriers. In a study done by Widyaman et al it was reported that the majority of the participants were able to correctly answer the questions regarding prevention of COVID-19 infection. ${ }^{2}$ In a previous study done among US dentists, it was found that $99.7 \%$ of the dental offices were using enhanced infection protection and control practices and many had also adopted advanced PPE. ${ }^{3}$

However, only $41 \%$ of the sample of dentists was confident to a considerable or great extent in handling suspected COVID-19 patients.

In the present study, majority of the study participants relied on information from a genuine source such as media $(91.3$ $\%$ ) for timely update on COVID-19 Infection. In a study done among dentists in Saudi Arabia, dental consultants and specialists showed higher awareness response of $90 \%$ and dentists with experience of eleven years or more showed $92.4 \%$ regarding the latest news on COVID-19 pandemic. $^{6}$ Many studies have shown similar results, where professionals with higher education and experience displayed higher awareness. ${ }^{8-11}$

Majority of the dentists in the current study perceived COVID-19 Infection as dangerous (98.4\%) and considered it to be a current serious public health issue and understood the importance of educating the public about COVID-19 to prevent the virus transmission. Almost two-third of the study sample believed that the COVID-19 symptoms self resolves over time and didn't require any special treatment. In a previous study, almost half of the dentists believed that the disease self resolves over time and didn't require any special treatment. ${ }^{1}$

The attitude of dentists regarding the treatment of patients during COVID-19 pandemic in their clinics varied. More than half of the study participants preferred to avoid working with a patient who is a suspect of COVID-19. In case a patient was coughing or sneezing, almost half of the dentists in the current study would refer the patient without treating him/her, $46.5 \%$ would treat the patient before referring to a hospital while the rest of the dentists would refuse the treatment and ask the patient to leave the dental clinic. In a similar study, it was reported that $43.8 \%$ would refer the patient to the hospital without treating them, $49.5 \%$ would treat the patient and refer them to the hospital and $4.6 \%$ would refuse the treatment. ${ }^{1}$

Majority of the dentists considered precautionary measures among the patients in the dental clinic as important during COVID-19 pandemic. They believed that asking the patients to maintain social distancing, to wear masks and perform hand hygiene as necessary in decreasing the COVID-19 transmission. In a similar study done in Saudi Arabia, it was found that almost two-third of the participants agreed that the dental reception area adopted the proper COVID-19 preventive measures. ${ }^{6}$ Furthermore, studies have shown that social distancing has been advocated as an important measure in preventing the COVID-19 disease transmission. $^{12}$

Most of the study participants were concerned about the safety of their dental staff and would not allow any of their dental team members to work with patients who had flu- like symptoms. Dentists in the present study considered the role of dentist in educating the public about COVID-19 as very significant. In a similar study, it was reported that almost all the dentists $(97.8 \%)$ knew about the importance of educating others about COVID-19 infection. ${ }^{1}$

In the current study, most of the dentists believed that they were prepared for the COVID-19 outbreak. Majority of the study participants knew whom to contact when an unprotected exposure occurs and what to do if they have signs and symptoms of the disease. However, when asked about the preparedness of their country, only $26 \%$ of the dentists gave a positive response.

Despite the findings of the current study, it is important to mention the limitations that were faced during the course of the survey, including the short period of data collection. This may have affected the sample size and the response rate of the study. Convenience sampling strategy was adopted which was not based on a random selection of sample. It has to be mentioned that the survey was conducted while the pandemic was still active. Furthermore, SARS-CoV2 infection is considered new and is not a fully understood or investigated topic. COVID-19 updates and recommendations are changed, modified or added frequently, hence the study focuses on where we currently stand and cannot be generalized.

Overall, the study has shown an acceptable level of awareness and preparedness among the dentists working in Bangalore city concerning the knowledge about COVID-19 infection, its transmission and measures for prevention. However, SARS-CoV-2 infection is a novel disease and is yet to be fully explored. Since the updates and recommendations about the disease are changed, modified or added frequently, the dentists have to make sure that they are up to date and well informed regarding the best practices and recommended disease management protocols. Educational sessions and training for the dentists adhering to the latest COVID-19 recommendations have to be carried 
out. Dental practitioners have to closely monitor their staffs and patients to ensure that they follow guidelines issued by the Ministry Of Health or the concerned authority. Lastly, adequate research has to be carried out to shed light on the unexplored areas regarding COVID-19 disease and infection control.

\section{ACKNOWLEDGEMENTS}

We sincerely thank all the participants of the study who spared a few minutes of their valuable time for filling the questionnaire.

\section{DECLARATIONS}

Funding: None

Conflict of interest: None declared

Ethical approval: Institutional ethical approval obtained

\section{REFERENCES}

I. Khader Y, Al Nsour M, Al-Batayneh OB, Saadeh R, Bashier H, Alfaqih M, Al-Azzam S. Dentists' awareness, perception, and attitude regarding COVID-19 and infection control: cross-sectional study among Jordanian dentists. JMIR public health and surveillance. 2020;6(2):e18798.

II. Widyarman AS, Bachtiar EW, Theodorea CF, Rizal MI, Roeslan MO, Djamil MS, Santosa DN, Bachtiar BM. COVID-19 Awareness Among Dental Professionals in Indonesia. Frontiers in medicine. 2020;7.

III. Estrich CG, Mikkelsen M, Morrissey R, Geisinger ML, Ioannidou E, Vujicic M, Araujo MW. Estimating COVID-19 prevalence and infection control practices among US dentists. The Journal of the American Dental Association. 2020 Nov 1;151(11):815-24.

IV. Cucinotta D, Vanelli M. WHO declares COVID-19 a pandemic. Acta Bio Medica: AteneiParmensis. 2020;91(1):157

V. Nasser Z, Fares Y, Daoud R, Abou-Abbas L. Assessment of knowledge and practice of dentists towards Coronavirus Disease (COVID-19): a cross- sectional survey from Lebanon. BMC oral health. 2020 Dec;20(1):1-9.

VI. Al-Khalifa KS, AlSheikh R, Al-Swuailem AS, Alkhalifa MS, Al-Johani MH, Al-Moumen SA, Almomen ZI. Pandemic preparedness of dentists against coronavirus disease: A Saudi Arabian experience. PloS one. 2020 Aug 19;15(8):e0237630.

VII. R. Dhanya, A.G Harikiran, V Deepthi. Dentist population ratio of Bangalore city: Adequate or inadequate-A descriptive study.jmscr. 2016 Dec;4(12):15048-54

VIII. Kamate SK, Sharma S, Thakar S, Srivastava D, Sengupta K, Hadi AJ, Chaudhary A, Joshi R, Dhanker K. Assessing Knowledge, Attitudes and Practices of dental practitioners regarding the COVID-19 pandemic: A multinational study. Dental and medical problems. 2020 Jan 1;57(1):11-7.

IX. Gupta N, Randhawa RK, Thakar S, Bansal M, Gupta $\mathrm{P}$, Arora V. Knowledge regarding Zika virus infection among dental practitioners of tricity area (Chandigarh, Panchkula and Mohali), India. Niger Postgrad Med J. 2016; 23(1):33-7

X. Gupta N, Mehta N, Gupta P, Arora V, Setia P. Knowledge regarding Ebola Hemorrhagic Fever among private dental practitioners in Tricity, India: A cross-sectional questionnaire study. Niger Med J. 2015; 56(2):138-42. https://doi.org/10.4103/03001652.153405 PMID: 25838631

XI. Holakouie-Naieni K, Ahmadvand A, Raza O, Assan A, Elduma AH, Jammeh A, et al. Assessing the Knowledge, Attitudes, and Practices of Students Regarding Ebola Virus Disease Outbreak. Iran J Public Health. 2015; 44(12):1670-6. PMID: 26811818

XII. Long RH, Ward TD, Pruett ME, Coleman JF, Plaisance MC. Modifications of emergency dental clinic protocols to combat COVID-19 transmission. Spec Care Dentist. 2020; 40(3):219-26. https://doi.org/ 10.1111/scd.12472 PMID: 32447777

Table 1. The characteristics of the study participants $(n=254)$

\begin{tabular}{|l|l|}
\hline Variable & Dentists, $\mathbf{n}(\%)$ \\
\hline Age (years) & \\
\hline$<30$ & $199(78.3)$ \\
\hline $30-39$ & $37(14.6)$ \\
\hline $40-49$ & $13(5.1)$ \\
\hline$\geq 50$ & $5(2)$ \\
\hline Gender & \\
\hline Female & $188(74)$ \\
\hline Male & $66(26)$ \\
\hline Years of practice & \\
\hline$\leq 5$ & $203(79.9)$ \\
\hline
\end{tabular}


Knowledge, Attitude and Perception Regarding COVID-19 Infection Control: A Cross-Sectional Study among Dentists in Bangalore City, India

\begin{tabular}{|l|l|}
\hline $6-10$ & $33(13)$ \\
\hline$>10$ & $18(7.1)$ \\
\hline Work place & \\
\hline Public sector & $28(11.02)$ \\
\hline Private clinic & $128(50.39)$ \\
\hline Institutions & $98(38.58)$ \\
\hline
\end{tabular}

Table 2. Awareness of the study participants $(n=254)$ about symptoms and modes of transmission of COVID-19 Infection

\begin{tabular}{|l|l|}
\hline Variable & Dentists, n (\%) \\
\hline Symptoms of COVID-19 Infection & \\
\hline Fever & $249(98)$ \\
\hline Cough & $249(98)$ \\
\hline Shortness of breath & $244(96.1)$ \\
\hline Sore throat & $242(95.3)$ \\
\hline No symptoms & $205(80.7)$ \\
\hline Diarrhoea & $172(67.7)$ \\
\hline Runny nose & $151(59.4)$ \\
\hline Joint/muscle pain & $133(52.4)$ \\
\hline Rash & $64(25.2)$ \\
\hline Red eyes & $54(21.3)$ \\
\hline Modes of transmission & \\
\hline Coughing and sneezing & $252(99.2)$ \\
\hline Hand shaking & $232(91.3)$ \\
\hline Touching surfaces such as door knobs and tables & $223(87.8)$ \\
\hline
\end{tabular}

Table 3. Awareness of the dentists $(n=254)$ regarding diagnostic tests, identification of patients at risk, high risk groups and measures for prevention of transmission of COVID-19 infection

\begin{tabular}{|l|l|}
\hline Variable & Dentists, n (\%) \\
\hline Diagnostic tests & $248(97.6)$ \\
\hline RT-PCR with respiratory material & $161(63.4)$ \\
\hline RT-PCR with serum sample & $105(41.3)$ \\
\hline Chest X- ray & \\
\hline $\begin{array}{l}\text { Identification of patients at risk of COVID-19 } \\
\text { infection }\end{array}$ & $218(85.8)$ \\
\hline History of travel to COVID-19 hit areas & $227(89.4)$ \\
\hline History of contact with possible infected patient & $241(94.9)$ \\
\hline Presence of symptoms of a respiratory infection & $95(37.4)$ \\
\hline Presence of symptoms of diarrhoea & $230(90.6)$ \\
\hline High risk groups & $239(94.1)$ \\
\hline Health care providers & $31(12.2)$ \\
\hline Elderly & $175(68.9)$ \\
\hline Male gender & $244(96.1)$ \\
\hline Children & $165(65)$ \\
\hline People with immune deficiency & $233(91.7)$ \\
\hline Travellers & $245(96.5)$ \\
\hline People with chronic diseases & $218(85.8)$ \\
\hline Measures for prevention of transmission & $252(99.2)$ \\
\hline $\begin{array}{l}\text { Frequently clean hands by using alcohol-based hand } \\
\text { rib or soap and water }\end{array}$ & \\
\hline Eat boiled and cooked food & \\
\hline Put face mask on known or suspected patients & \\
\hline Place known or suspected patients in adequately & \\
\hline & $265.7)$ \\
\hline
\end{tabular}


Knowledge, Attitude and Perception Regarding COVID-19 Infection Control: A Cross-Sectional Study among Dentists in Bangalore City, India

\begin{tabular}{|l|l|}
\hline ventilated single rooms & $243(95.7)$ \\
\hline All health staff members wear protective clothing & $221(87)$ \\
\hline $\begin{array}{l}\text { Avoid moving and transporting patients out of their } \\
\text { area unless necessary }\end{array}$ & $244(96.1)$ \\
\hline $\begin{array}{l}\text { Routinely clean and disinfect surfaces in contact with } \\
\text { known or suspected patients }\end{array}$ & \\
\hline
\end{tabular}

Table 4. Sources of information of the study participants $(n=254)$ about COVID-19 infection

\begin{tabular}{|l|l|}
\hline $\begin{array}{l}\text { Sources of information of the dentists on COVID- } \\
\mathbf{1 9}\end{array}$ & Dentists, $\mathbf{n}(\boldsymbol{\%})$ \\
\hline Media (newspaper, television, radio etc.) & $232(91.3)$ \\
\hline Social network (Facebook, twitter etc.) & $194(76.4)$ \\
\hline Friends or family member & $130(51.2)$ \\
\hline Medical doctors or routinely visited physicians & $131(51.6)$ \\
\hline Academic training courses & $87(34.3)$ \\
\hline Colleagues & $134(52.8)$ \\
\hline Government organization such as Ministry of Health & $168(66.1)$ \\
\hline
\end{tabular}

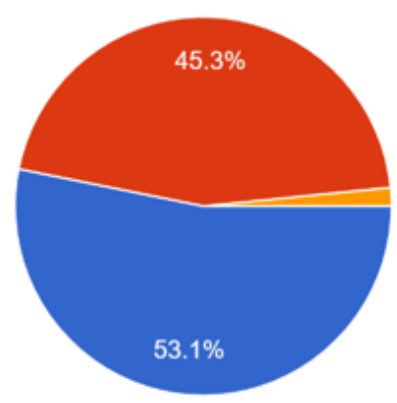

Very dangerous

Moderately dangerous

Not dangerous

Fig 1. Distribution of study participants according to how they perceived COVID-19

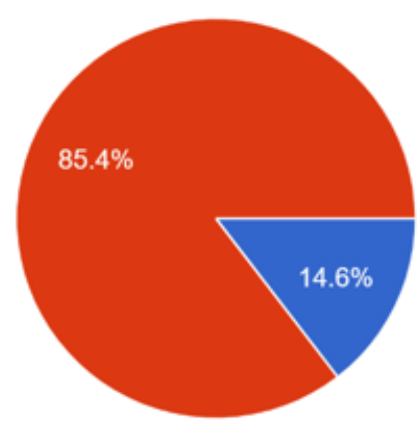

Fig 2. Distribution of study participants when asked whether they considered COVID-19 as a serious public health issue

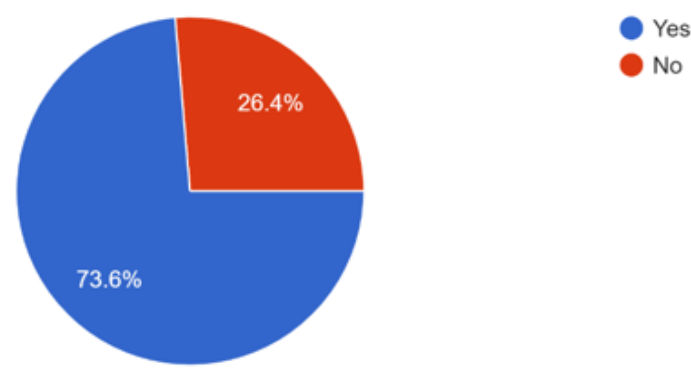

Fig 3. Distribution of study participants according to whether they considered themselves prepared for COVID-19 outbreak 